\title{
Intestinal injury following liver transplantation was mediated by TLR4/NF-кB activation-induced cell apoptosis
}

\author{
DONG-DONG YUAN ${ }^{1 *}, \mathrm{XIN}-J I N ~ C H I^{1^{*}}, \mathrm{YI} \mathrm{JIN}^{2}, \mathrm{XI} \mathrm{LI}^{3}$, MIAN GE$^{1}$, WAN-LING GAO ${ }^{1}$, \\ JIAN-QIANG GUAN ${ }^{1}$, AI-LAN ZHANG ${ }^{1}$ and ZI-QING HEI ${ }^{1}$ \\ Departments of ${ }^{1}$ Anesthesiology, ${ }^{2}$ Pathology and ${ }^{3}$ Thyroid and Breast Surgery, \\ The Third Affiliated Hospital of Sun Yat-sen University, Guangzhou, Guangdong 510630, P.R. China
}

Received February 3, 2015; Accepted November 30, 2015

DOI: $10.3892 / \mathrm{mmr} .2015 .4719$

\begin{abstract}
Intestinal motility and barriers are often impaired due to intestinal congestion during liver transplantation. Intestinal bacteria and enterogenous endotoxins enter into the blood stream or lymphatic system and translocate to other organs, which can result in postoperative multi-organ dysfunction (MODF) and systemic inflammatory reaction syndrome (SIRS) severely affecting patient survival. However, the mechanisms underlying liver transplantation-induced intestinal injury remain unclear and effective therapies are lacking. Thus, the present study investigated whether these effects were associated with endotoxin-mediated apoptosis. Rat autologous orthotopic liver transplantation (AOLT) models were established to observe dynamic intestinal injuries at different time-points following reperfusion. Changes in the levels of endotoxins and the primary receptor, toll-like receptor 4 (TLR4), as well as its downstream signaling molecule, nuclear factor $-\kappa \mathrm{B}(\mathrm{NF}-\kappa \mathrm{B})$ were all determined. Finally, immunohistochemistry and terminal deoxynucleotidyl transferase dUTP nick end labeling assays were conducted to detect caspase-3 expression and intestinal cell apoptosis, respectively. AOLT resulted in significant pathological intestinal injury, with the most serious intestine damage apparent four or eight hours following reperfusion. Furthermore, the levels of endotoxins and inflammatory cytokines, such as tumor necrosis factor- $\alpha$ and interleukin-6, peaked during this time period and gradually decreased to the normal level. Notably, TLR4 and downstream $\mathrm{NF}-\kappa \mathrm{B}$ expression, as well as NF- $\mathrm{B}$-mediated caspase-3
\end{abstract}

Correspondence to: Professor Xin-Jin Chi or Professor Zi-Qing Hei, Department of Anesthesiology, The Third Affiliated Hospital of Sun Yat-sen University, 600 Tianhe Road, Guangzhou, Guangdong 510630, P.R. China

E-mail: chixinjin@yeah.net

E-mail: heiziqing@sina.com

\section{*Contributed equally}

Key words: liver transplantation, intestinal injury, endotoxin, toll-like receptor 4 , nuclear factor- $\kappa \mathrm{B}$, apoptosis activation and intestinal cell aapoptosis coincided with the intestinal pathological damage. Thus, the possible mechanism of post-liver transplantation intestinal injury was demonstrated to be associated with $\mathrm{NF}-\kappa \mathrm{B}$ activation-induced cell apoptosis.

\section{Introduction}

Liver transplantation is a serious surgical procedure, which can result in complications to a number of other organs $(1,2)$. Multi-organ dysfunction (MODF) and systemic inflammatory reaction syndrome (SIRS) are the two main complications following liver transplantation, and contribute to a high patient mortality rate; however, the underlying mechanisms of these complications remain to be determined (3). During liver transplantation, intestinal congestion is inevitable due to inferior vena cava (IVC) and portal vein (PV) interruption, which results in intestinal motility disorders and destruction of intestinal barriers (4). As previously reported, intestinal barriers are a complex system and perform two important functions in the body; nutrient absorption and defence against harmful macromolecule penetration. Intestinal barriers are composed of physical, chemical, biological and immunological elements. The physical aspect includes a mucous layer, intestinal epithelial cells and tight junctions located at the apical surface. The chemical barrier involves gastric acid, digestive enzymes and bile. The immunological barrier refers to lymphocytes and immunoglobulin A (IgA) and the biological barrier is composed of normal intestinal flora, and the important environmental factors for energy absorption and storage. Destruction of the intestinal barriers presents in a variety of ways, including flora shift, small intestinal bacterial overgrowth, tight junction alterations and increased gut permeability (5). Enterogenous endotoxins are over-produced and there is increase in bacterial translocation (6). Once intestinal bacteria or endotoxins enter the venous or lymphatic system, they translocate to other organs and result in remote organ damage $(7,8)$. Thus, intestinal epithelial cell protection is important for patients undergoing liver transplantation, and may be an effective strategy to protect against MODF and SIRS. Therefore, there is an urgent requirement to investigate the mechanisms underlying intestinal injury and to develop effective strategies to protect against this damage. 
Endotoxins are one of the most important constituents of the outer membrane of Gram-negative bacteria (GNB) and are key to the pathogenesis of GNB-associated MODF and SIRS (9). Endotoxins that are over-produced during liver transplantation bind to the primary receptor, toll-like receptor 4 (TLR4), which is important in post-liver transplantation intestinal injury $(10,11)$. TLR4 predominantly located on cell membranes recognizes pathogen-associated molecular patterns. When activated during an infection, it induces the transcription of certain immune genes and results in activation of the nuclear factor (NF)- $\mathrm{B}$ signaling pathway and downstream inflammatory cascade that is activated by inflammatory mediators, such as tumor necrosis factor (TNF)- $\alpha$ and interleukin (IL)-6 (12).

$N F-\kappa B$ is the final effector molecule of the TLR4 signaling pathway and is pivotal in the translation and transcription of inflammatory mediators and caspase expression, which promotes the development of numerous intestinal diseases (13). Overproduction of pro-inflammatory cytokines is a characteristic of patients that have undergone liver transplantation. In addition, apoptosis is considered to be important in injuries of remote organs following liver transplantation $(14,15)$. Thus, the present study aimed to determine whether apoptosis mediated by the overproduction of inflammatory cytokines, via TLR4/NF- $\mathrm{B}$ signal pathway activation, may be the potential mechanism underlying intestinal injury.

\section{Materials and methods}

Animals. All the experiments were conducted according to the National Institutes of Health criteria for the care and use of laboratory animals in research. The study was approved by the Laboratory Animal Care Committee of Sun Yat-sen University (Guangzhou, China). Male Sprague-Dawley rats (age, 8 weeks; weight, 200-220 g) were purchased from the Laboratory Animal Center of Sun Yat-sen University and randomly assigned into five parallel groups using a random number table and taking into consideration the weight of the rats. The groups were as follows: Sham, and reperfusion 4, 8 , 16 and $24 \mathrm{~h}$ (AOLT model) groups ( $\mathrm{n}=8$ per group). The rats (four per cage) were housed at room temperature, fed with standard rat chow and had access to tap water ad libitum. The room was well-ventilated with a 12-h light/dark cycle. The rats were acclimated for one week prior to experiments. Food was withheld $8 \mathrm{~h}$ prior to commencing the experiments, however, all animals had free access to water.

Rat autologous orthotopic liver transplantation (AOLT) model establishment. This model was established according to the methods of previous studies $(3,12)$. Briefly, an open face guard was used to administer the ether inhalational anesthesia (Shanghai Baxter Healthcare Co., Ltd., Shanghai, China) until the rats exhibited no response to a needle stimulus. An incision was made to open the abdominal cavity, the liver falciform ligament was ligated and the blood vessel along the esophagus was severed. Then, the liver was exposed. Following liberation of the supra hepatic vena cava (SVC), the liver was replaced in its original position. The IVC was then dissociated, until the upper region of the left renal vein was completely liberated. The first hepatic portal (the H-shaped groove on the surface of the liver, from which the portal vein, hepatic duct and hepatic artery access the liver) was also dissected and the PV was separated from the convergence of the inferior mesenteric and splenic veins. The hepatic artery and biliary tract were also successively detached, according to their anatomic relationship, and the first hepatic portal was ligated Microvascular clamps were used at the convergence of the inferior mesenteric, splenic veins, hepatic artery, SVC and IVC, and the PV was punctured with a 24-gauge needle in preparation for reperfusion. Then, a 1-mm incision was made in the IVC wall as an outflow tract and $2.5 \mathrm{ml} / \mathrm{min}$ pre-cold $4^{\circ} \mathrm{C}$ Ringer's lactate solution (Shanghai Baxter Healthcare Co., Ltd.,) was injected until the liver turned yellow. Finally, the incisions in the PV and IVC were closed using 8-0 sutures (Hangzhou Huawei Medical Appliance Co., Ltd., Hangzhou, China). PV, SVC, IVC and the hepatic artery were all unclamped. The duration of the anhepatic phase was $20 \pm 1$ min (1).

The sham group underwent abdominal surgery and liver dissection under ether inhalational anesthesia without cold perfusion or reperfusion. The AOLT model rats were subjected to the typical pathophysiological hepatic ischemia/reperfusion $(\mathrm{I} / \mathrm{R})$ processes during liver transplantation. Compound lidocaine cream (Beijing Ziguang Pharmaceutical Co., Ltd., Beijing, China) was applied to the incisions for pain relief. Following surgery, the rats were maintained in a temperaturecontrolled environment under a 12-h light/dark cycle with free access to water. Rats in the sham group were sacrificed by cervical dislocation $8 \mathrm{~h}$ after surgery and the samples were subsequently obtained. Rats in the AOLT groups were sacrificed by cervical dislocation $4,8,16$ and $24 \mathrm{~h}$ after reperfusion and the samples were obtained at the corresponding time-points.

Histopathological examination. Intestinal specimens were obtained from $5 \mathrm{~cm}$ above the terminal ileum and fixed in $10 \%$ buffered formalin (Nanjing KeyGen Biotech Co., Ltd., Nanjing, China), embedded in paraffin (Nanjing Keygen Biotech Co., Ltd.), and processed for hematoxylin and eosin staining (Nanjing Keygen Biotech Co., Ltd.). Samples were visualized under an Eclipse E800 light microscope (Nikon Corporation, Tokyo, Japan) and intestinal mucosal damage was graded according to Chiu's criteria (3): Grade 0, normal mucosa villi; grade 1, development of subepithelial Gruenhagen's space at the tip of the villi; grade 2, extension of the subepithelial space with moderate epithelial lifting; grade 3, extensive epithelial lifting, possibly with a few denuded villi; grade 4, denuded villi with dilated capillaries, and increased cellularity of the lamina propria and exposed capillaries; and grade 5, disintegration of the lamina propria, ulceration and hemorrhage.

Assessment of fatty acid-binding protein 2 (FABP2), diamine oxidase (DAO), lipopolysaccharide (LPS), TNF- $\alpha$ and IL-6. Plasma was harvested from the collected abdominal aortic blood (2-ml blood samples were collected from each rat in the sham group $8 \mathrm{~h}$ after surgery, and from the AOLT model groups at $4,8,16$ and $24 \mathrm{~h}$ post-reperfusion) and maintained at $-20^{\circ} \mathrm{C}$. The levels of DAO, FABP2, TNF- $\alpha$ and IL- 6 were determined using their corresponding enzyme-linked immunosorbent assay (ELISA) kits according to the manufacturer's protocol 
A

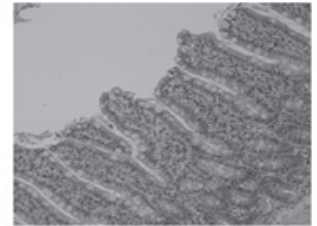

Sham

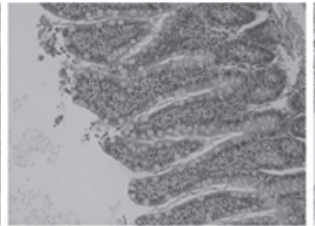

$4 \mathrm{~h}$

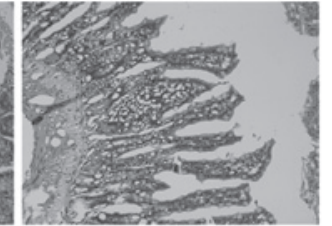

$8 \mathrm{~h}$

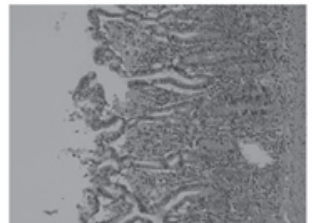

$16 \mathrm{~h}$

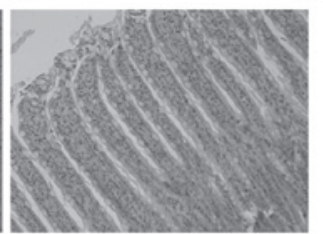

$24 \mathrm{~h}$
B

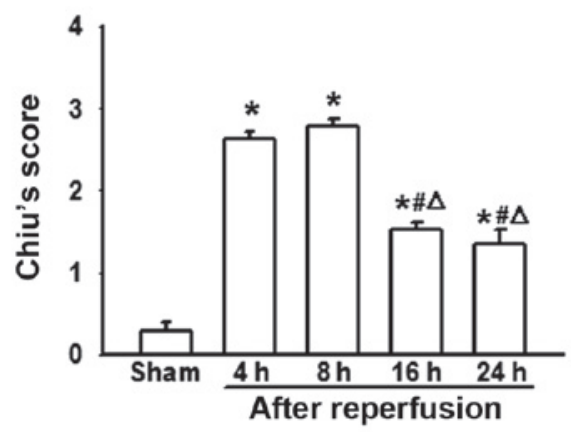

C

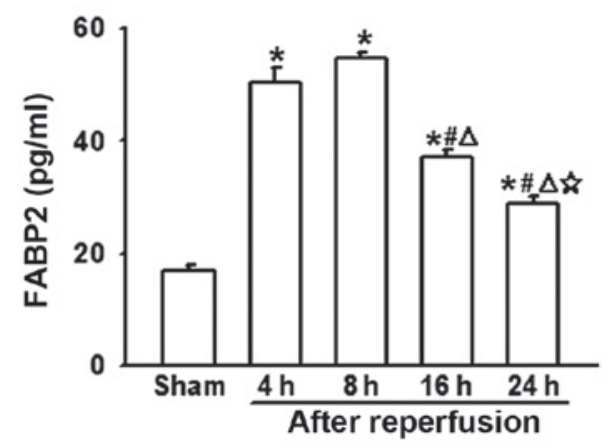

D

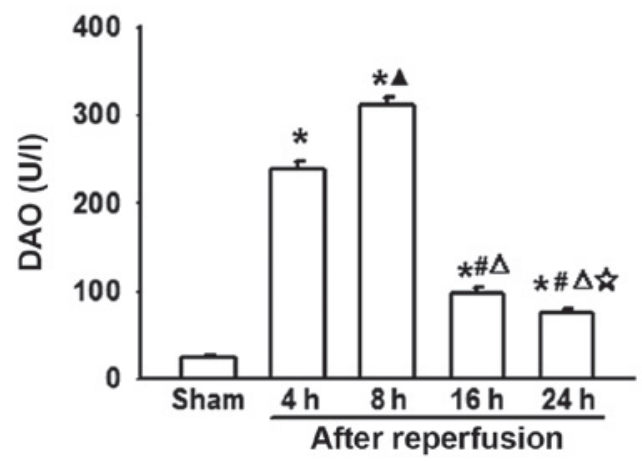

Figure 1. Intestine injury of rats following autologous orthotopic liver transplantation at different time-points. (A) Intestinal injury of rats in the different groups, as demonstrated by hematoxylin and eosin staining. Magnification, x200. (B) Chiu's score of the intestinal mucosa in the different groups. Levels of (C) FABP2 and (D) DAO in the different groups. Each group contains 8 samples. ${ }^{*} \mathrm{P}<0.05$ vs. the sham group; ${ }^{*} \mathrm{P}<0.05$ vs. the $4 \mathrm{~h}$ after reperfusion group; ${ }^{\Delta} \mathrm{P}<0.05$ vs. the $8 \mathrm{~h}$ after reperfusion group; $\mathrm{P}<0.05$ vs. the $16 \mathrm{~h}$ after reperfusion group. FABP2, fatty acid-binding protein 2 ; DAO, diamine oxidase.

(all kits were obtained from R\&D Systems, Minneapolis, MN, USA). LPS was detected using an endotoxin detection assay kit (Lonza, Basel, Switzerland).

Western blotting. Western blotting was conducted as described previously $(3,12)$. Briefly, the membranes were blocked for $30 \mathrm{~min}$ at room temperature with $5 \%$ non-fat dry milk (Sigma-Aldrich, St. Louis, MO, USA) and immunoblotted using anti-TLR4 (cat. no. sc-293072; monoclonal mouse anti-rat; 1:1,000; Santa Cruz Biotechnology, Inc., Dallas, TX, USA) overnight at $4^{\circ} \mathrm{C}$. Following several washes, the membranes were incubated for $1 \mathrm{~h}$ at room temperature with horseradish peroxidase (HRP)-conjugated polyclonal goat anti-mouse IgG (cat. no. sc-2005; 1:2,000; Santa Cruz Biotechnology, Inc.) to detect TLR4 expression. Anti-glyceraldehyde 3-phosphate dehydrogenase (GAPDH; cat. no. sc-47724; monoclonal mouse anti-rat; 1:1,000; Santa Cruz Biotechnology, Inc.) and its corresponding secondary antibody, HRP-conjugated polyclonal goat anti-mouse IgG (1:2,000; Santa Cruz Biotechnology, Inc.) served as a control. Protein-antibody complexes were detected with an enhanced chemiluminescence system (Keygen Biotech Co., Ltd.). Protein band sizes were estimated using AlphaView 2.2.14407 software (ProteinSimple, Santa Clara, CA, USA). The density was correlated to the protein expression and normalized to GAPDH.
Immunofluorescence. Immunofluorescent staining was conducted according to the appropriate protocol as previously described (1). Cryostat sections of OCT-embedded intestinal samples $(4-\mu \mathrm{m})$ were incubated with primary antibodies against P65 (cat. no. sc-8008; monoclonal mouse anti-rat; 1:100; Santa Cruz Biotechnology, Inc.) for one night at $4^{\circ} \mathrm{C}$. Following incubation with goat anti-mouse IgG-fluorescein isothiocyanate (FITC; cat. no. sc-2010; 1:100; Santa Cruz Biotechnology, Inc.), the sections were observed and imaged under x400 magnification using a fluorescence microscope (Olympus BX40; Olympus Corporation, Tokyo, Japan). Nuclei were stained with 4',6-diamidino-2-phenylindole $(1 \mu \mathrm{g} / \mathrm{ml}$; Nanjing KeyGen Biotech Co., Ltd.).

Immunohistochemistry. Immunohistochemical staining was performed in 4- $\mu \mathrm{m}$ paraffinized sections, as described previously (1). After being dewaxed and dehydrated, the sections were incubated with $\mathrm{H}_{2} \mathrm{O}_{2}$ (3\%; Nanjing Keygen Biotech Co., Ltd.) in order to inhibit endogenous peroxidase activity. The slides were incubated with primary antibodies against caspase-3 (cat. no. sc-7148; polyclonal rabbit anti-rat; 1:100; Santa Cruz Biotechnology, Inc.) overnight at $4^{\circ} \mathrm{C}$. Following incubation with the corresponding secondary antibody, goat anti-rabbit IgG-FITC (cat. no. sc-2012; 1:100; Santa Cruz Biotechnology, Inc.), the samples were visualized under a light microscope (Eclipse E800). Five photomicrographs were captured randomly 
A

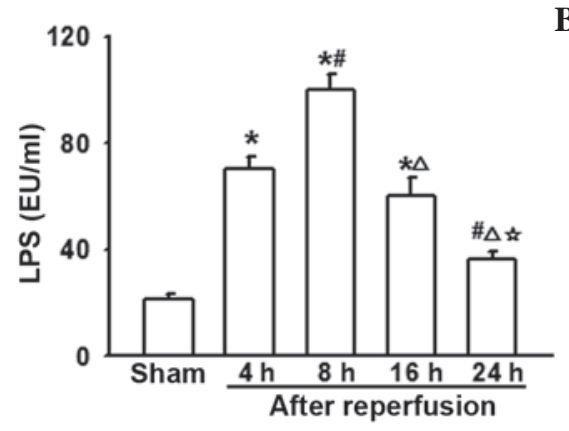

B

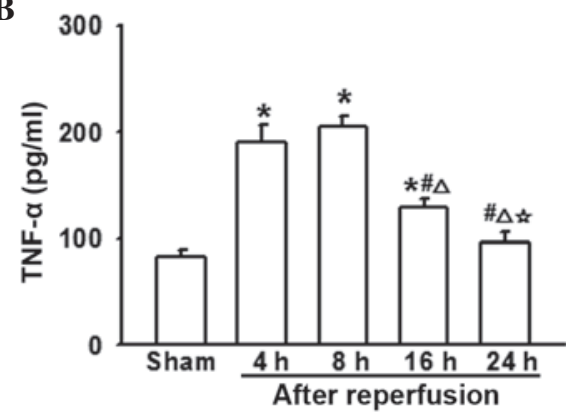

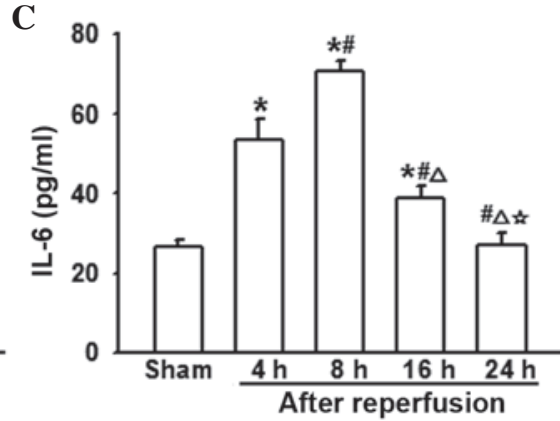

Figure 2. Levels of LPS, TNF- $\alpha$ and IL-6 following AOLT at different time-points. Changes of (A) LPS, (B) TNF- $\alpha$ and (C) IL-6 expression at different reperfusion time-points after AOLT. Each group contains eight samples. ${ }^{*} \mathrm{P}<0.05$ vs. the sham group; ${ }^{*} \mathrm{P}<0.05$ vs. the $4 \mathrm{~h}$ after reperfusion group; ${ }^{\Delta} \mathrm{P}<0.05$ vs. the $8 \mathrm{~h}$ after reperfusion group; $\mathrm{P}<0.05 \mathrm{vs}$. the $16 \mathrm{~h}$ after reperfusion group. LPS, lipopolysaccharide; TNF, tumor necrosis factor; IL, interleukin; AOLT, autologous orthotopic liver transplantation.

$\mathbf{A}$
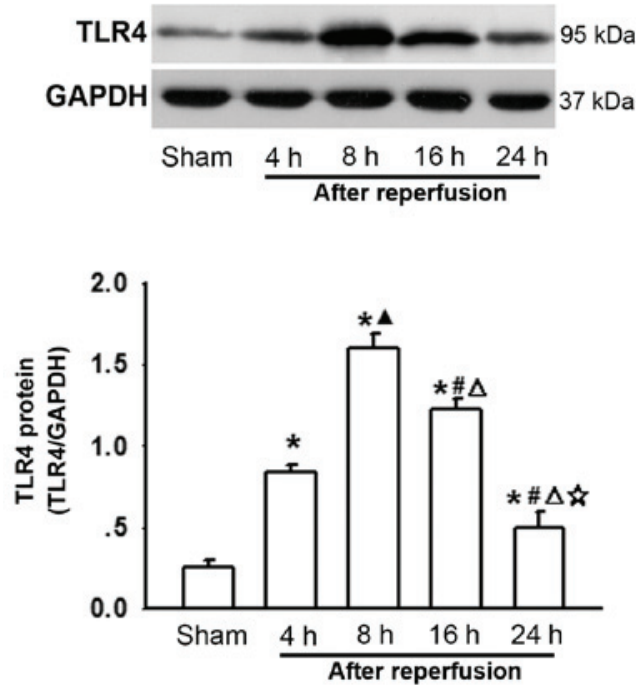

B

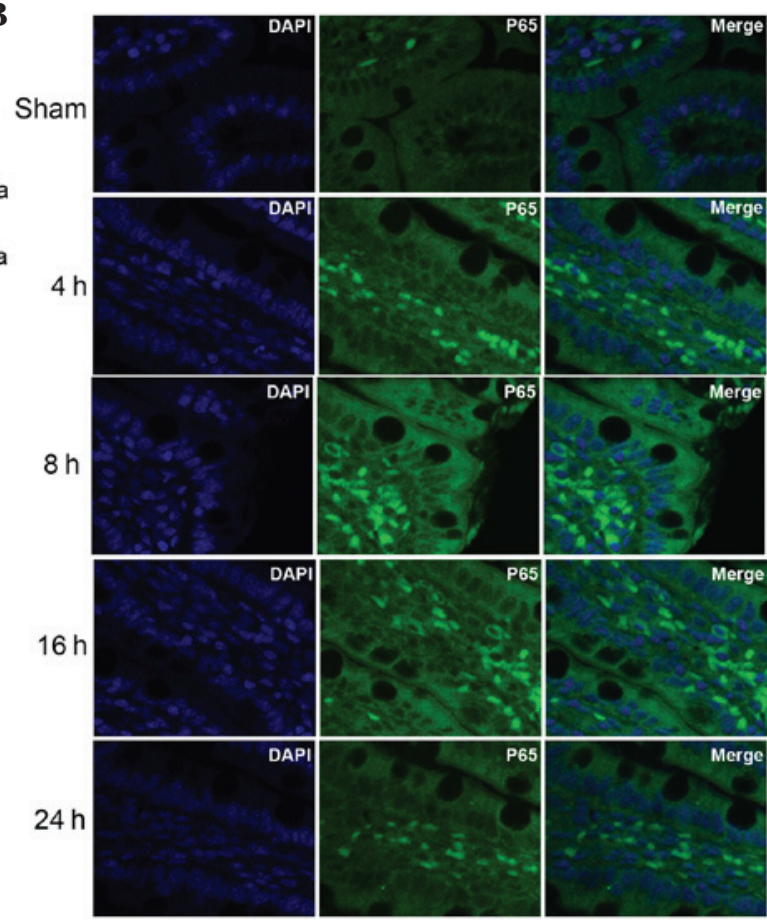

Figure 3. Changes of TLR4 and P65 expression following autologous orthotopic liver transplantation at different time-points. (A) Changes in TLR4 expression as determined by western blotting. (B) Changes in P65 expression as determined by a fluorescence microscope and imaged under x400 magnification (stain, DAPI). Each group contains eight samples. ${ }^{*} \mathrm{P}<0.05$ vs. the sham group; ${ }^{*} \mathrm{P}<0.05$ vs. the $4 \mathrm{~h}$ after reperfusion group; ${ }^{\Delta} \mathrm{P}<0.05$ vs. the $8 \mathrm{~h}$ after reperfusion group; $\mathrm{P}<0.05$ vs. the $16 \mathrm{~h}$ after reperfusion group. TLR4, toll-like receptor 4; GAPDH, glyceraldehyde 3-phosphate dehydrogenase; DAPI, 4',6-diamidino-2-phenylindole.

(magnification, x400). The average optical density (AOD) of the photomicrographs was quantified using the high resolution pathological image analysis system (HPIAS-1000; (Nanjing Keygen Biotech Co., Ltd.) to measure expression levels of caspase-3.

Terminal deoxynucleotidyl transferase dUTP nick end labeling (TUNEL) assay. The TUNEL assay (Nanjing Keygen Biotech Co., Ltd.) was conducted to evaluate intestinal tissue apoptosis according to the manufacturer's instructions. Briefly, 4- $\mu \mathrm{m}$ paraffinized sections were dewaxed, rehydrated, and incubated with terminal deoxynucleotidyl transferase enzyme at $37^{\circ} \mathrm{C}$ for $1 \mathrm{~h}$. The reaction was detected by incubation with anti-digoxigenin-peroxidase (Nanjing Keygen Biotech
Co., Ltd.) for $30 \mathrm{~min}$ at room temperature and visualized in a buffer containing 3,3'diaminobenzidine. The samples were counterstained by immersion in hematoxylin. The stained tissue samples were observed using a fluorescence microscope (EclipseE800) and five photomicrographs were obtained randomly (magnification, $x 400$ ). The AOD from the photomicrographs was quantified using the high-resolution pathological image analysis system (HPIAS-1000) to measure TUNEL-positive cells.

Statistical analysis. Statistical analysis was performed using SPSS 15.0 software (SPSS, Inc., Chicago, IL, USA). Multiple comparisons among groups were analyzed using one-way analysis of variance, followed by Tukey's post hoc 
A

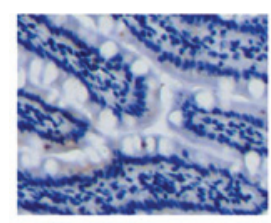

Sham

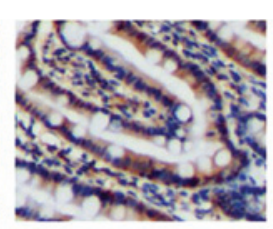

$4 \mathrm{~h}$

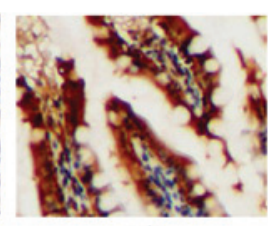

$8 \mathrm{~h}$

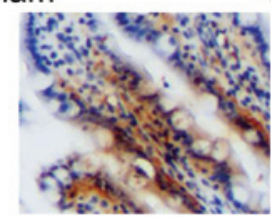

$16 \mathrm{~h}$

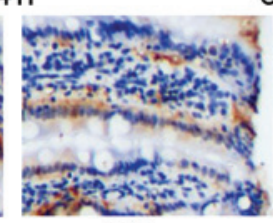

$24 \mathrm{~h}$

C

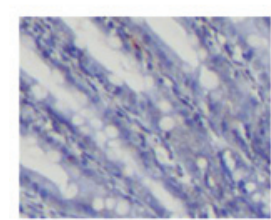

Sham

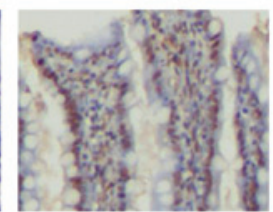

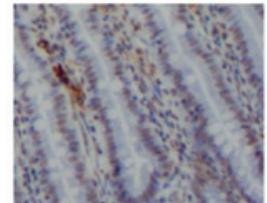

$16 \mathrm{~h}$ $4 \mathrm{~h}$

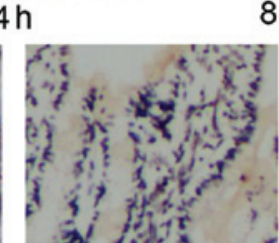

$24 \mathrm{~h}$
B

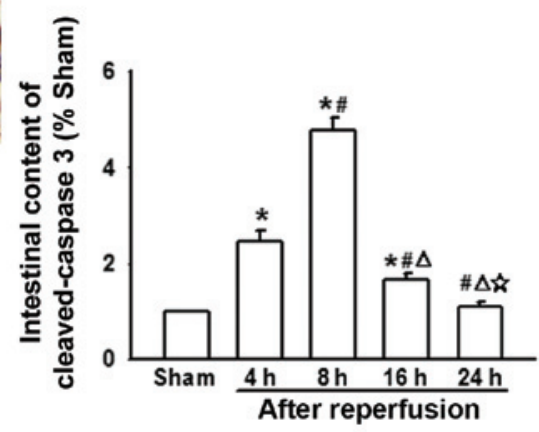

D

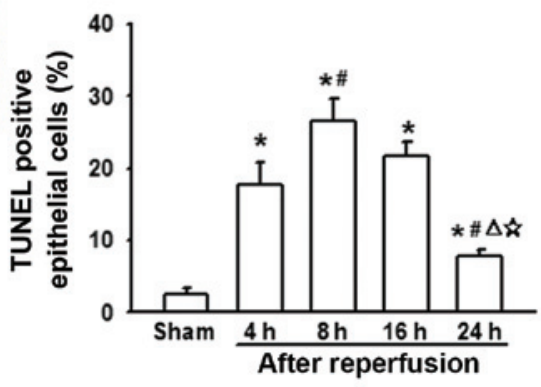

Figure 4. Immunohistochemistry staining of caspase-3 and intestinal epithelial cell apoptosis following AOLT at different time-points. (A) Changes of TLR4 expression at different reperfusion time-points after AOLT, using immunohistochemistry staining. The immunostained tissue samples were visualized and photographed under a light microscope (magnification, x400). (B) Five photomicrographs were taken from five high-power fields randomly for each sample. AOD from the photomicrographs was then quantified to measure caspase-3 expression. (C) Intestinal epithelial cell apoptosis at different reperfusion time-points after AOLT was observed using TUNEL staining (magnification, x400). (D) In very sample, five photomicrographs were taken from five high-power fields randomly. AOD from the photomicrographs was then quantified to measure TUNEL expression. Each group contains eight samples. * $\mathrm{P}<0.05 \mathrm{vs}$. the sham group; ${ }^{\#} \mathrm{P}<0.05$ vs. the $4 \mathrm{~h}$ after reperfusion group; ${ }^{\Delta} \mathrm{P}<0.05$ vs. the $8 \mathrm{~h}$ after reperfusion group; $\mathrm{P}<0.05$ vs. the $16 \mathrm{~h}$ after reperfusion group. AOLT, autologous orthotopic liver transplantation; TLR4, toll-like receptor 4; AOD, average optical density; TUNEL, terminal deoxynucleotidyl transferase dUTP nick end labeling.

comparisons. $\mathrm{P}<0.05$ was considered to indicate a statistically significant difference.

\section{Results}

Intestinal injuries following AOLT at different time-points. In the present study, rat AOLT models were established to investigate the effect of liver transplantation on the intestines. In this model, IVC and PV interruption during the anhepatic phase leads to blood reflux disorder and serious intestinal congestion, which result in significant intestinal injuries (3). In the present experiments, it was demonstrated that as the reperfusion time was extended, pathological damage to the intestines increased. Four or eight hours after reperfusion, intestinal damage peaked, resulting in extensive epithelial lifting from the villi, which recovered gradually (Fig. 1A and B). It was also demonstrated that FABP2 and DAO levels were notably increased $8 \mathrm{~h}$ following reperfusion, which mirrored the pathological injury of the intestine (Fig. 1C and D).

Changes in the levels of LPS, TNF- $\alpha$ and IL-6 following AOLT at different time-points. Fig. 1 illustrated the significance of AOLT-mediated intestinal injury. However, its mechanism remains unclear. Intestinal motility and barriers have been shown to be impaired following liver transplantation, resulting in increased bacterial translocation and enterogenous endotoxin levels $(3,16)$. This was shown to initiate the overproduction of pro-inflammatory cytokines, which may trigger remote organ injury (3). The present study demonstrated that the level of LPS increased significantly 4-8 h following reperfusion and then decreased gradually (Fig. 2A). TNF- $\alpha$ and IL-6 are important inflammatory factors in liver transplantation and are key in various inflammatory reactions. Thus, the levels of TNF- $\alpha$ and IL- 6 were determined in the present study. Fig. 2 shows that $4-8 \mathrm{~h}$ following reperfusion, the levels of TNF- $\alpha$ and IL-6 were significantly increased, compared with the sham group and then declined gradually. After 24 h, no significant difference was identified between sham group and the AOLT model groups (Fig. 2B and C).

Changes in TLR4 and P65 expression on the surface of intestine cells following AOLT at different time-points. To the best of our knowledge, intestinal congestion in liver transplantation always results in enterogenous endotoxin over-production and an increase in the levels of inflammatory cytokines, which contribute to remote organ injury (Figs. 1 and 2). LPS activates TLR4 and its downstream NF- $\kappa \mathrm{B}$, and TNF- $\alpha$ triggers translocation of $N F-\kappa B$ to the nucleus (17), thus changes in TLR4 and 
P65 expression were investigated. As shown in Fig. 3A, TLR4 protein levels were increased after AOLT and peaked $8 \mathrm{~h}$ after reperfusion, which coincided with the most severe intestinal pathological damage (Fig. 1) and the increase in LPS, TNF- $\alpha$ and IL-6 levels (Fig. 2). In addition, P65 was activated at this time-point (Fig. 3B). Subsequently, the expression of TLR4 and P65 gradually decreased as reperfusion time increased (Fig. 3A and B). These results suggested that the TLR4/NF- $\kappa \mathrm{B}$ signaling pathway participated in post-AOLT intestinal injury.

Changes in caspase-3 and apoptosis levels in the intestine following AOLT at different time-points. During liver transplantation, intestinal congestion was severe due to IVC and PV interruption. This lead to enterogenous endotoxin and inflammatory cytokine over-production, which could activate the TLR4/NF- $\kappa$ B signaling pathway (Figs. 2 and 3). Previous studies have demonstrated that cytokine over-production and $\mathrm{TLR} 4 / \mathrm{NF}-\kappa \mathrm{B}$ signal pathway activation triggers caspase-3-independent cell apoptosis $(18,19)$. Thus, it was hypothesized that this may be the reason for liver transplantation-mediated intestinal injury. The present results indicated that expression of caspase- 3 also peaked $8 \mathrm{~h}$ following reperfusion, which was in line with the levels of LPS, TNF- $\alpha$, IL-6, TLR4/P65, and also decreased in line with the reduction in the levels of LPS, TNF- $\alpha$, IL-6 and TLR4/P65 (Fig. 4A). In addition, the levels of TUNEL-positive epithelial cells coincided with caspase-3 expression (Fig. 4B). This suggests that caspase-3-induced apoptosis mediated by cytokines and the TLR4/NF- $\mathrm{B}$ signaling pathway may be important in post-liver transplantation intestinal injury.

\section{Discussion}

Liver transplantation is considered to be the most effective strategy to cure final-stage liver disease; however, it is associated with a number of serious complications that affect patient survival $(1,2)$. It has previously been demonstrated that intestinal motility and barriers are impaired following liver transplantation (20). Findings from the present study (Fig. 1) demonstrated that pathological injury of the intestine was most marked $8 \mathrm{~h}$ after reperfusion, which was coincident with the changes in FABP2 and DAO levels (well-known markers of intestinal mucosa barrier destruction). Bacterial translocation and enterogenous endotoxemia are notable, and may lead to post-operative MODF and SIRS (21). These contribute to the high mortality rate of patients undergoing liver transplantation; however, the mechanism underlying these effects remains unclear. Thus, in the present study, rat AOLT models were established to investigate the possible mechanisms underlying liver transplantation-induced intestinal injury. The rat AOLT model has advantages compared with allogeneic orthotopic liver transplantation, such as avoiding obvious rejection reactions and complex conditions of recipients, in addition there is repeatability of experiments and a high survival rate of rats. Compared with a pure hepatic I/R injury model, rat AOLT models undergo the predominant procedures involved in liver transplantation, including blood reflux disorder (1). Thus, the rat AOLT model was used in the present study.

During liver transplantation, IVC and PV interruption result in severe intestinal congestion. In addition, intestinal motility and barriers have been shown to be impaired following liver transplantation (20). Bacterial translocation and enterogenous endotoxin increase, which result in enterogenous LPS over-production, inflammatory cytokine production (TNF- $\alpha$ and IL-6) and TLR4/NF- $\kappa \mathrm{B}$ signaling pathway activation $(17,21,22)$. The present study determined that the TLR4/NF- $\kappa \mathrm{B}$ signal pathway activation triggered caspase-3-independent cell apoptosis. Caspase-3 expression and intestinal epithelial cell apoptosis were notably increased following AOLT. These changes coincided with pathological damage of the intestine following liver transplantation. This indicated that post-liver transplantation intestinal injury was mediated by endotoxin over-production, cytokine production and TLR4/NF- $\mathrm{B}$ signaling pathway-induced apoptosis.

Bacterial translocation and enterogenous endotoxin are important in remote organ damage (23). LPS is one of the most important constituents of the outer membrane of GNB and is recognized to be key in the pathogenesis of GNB-associated sepsis $(24,25)$. The present study demonstrated that the levels of enterogenous endotoxin peaked at $8 \mathrm{~h}$ after reperfusion, the point at which the intestine exhibited the most notable damage (Fig. 2A). Macromolecular LPS binds to TLR4 (considered to be the primary receptor), which results in myeloid differentiation primary response gene 88-dependent signaling and activation of the downstream inflammatory cascade. Furthermore, activation of I $\mathrm{B}$ kinase- $\beta$ and mitogen-activated protein kinase phosphorylation were important in this process $(18,26)$. This mediates NF- $\kappa \mathrm{B}$-dependent nuclear transcription and TNF- $\alpha$ production.

During liver transplantation, IVC and PV interruption lead to liver I/R injury, which initiates the over-production of pro-inflammatory cytokines $(3,22)$. TNF- $\alpha$ is considered to be an important pro-inflammatory cytokine, which mediates the degradation of $\mathrm{I} \kappa \mathrm{B} \alpha$ and promotes translocation of $\mathrm{NF}-\kappa \mathrm{B}$ to the nucleus (27). Simultaneous over-production of LPS/TLR4/NF- $\mathrm{B}$-induced inflammatory cytokine along with over-production of pro-inflammatory cytokines triggered remote organ injuries. TNF- $\alpha$ is not a direct chemoattractant, however, following liver transplantation, it induced inflammation via the $\mathrm{NF}-\kappa \mathrm{B}$ signaling pathway. TNF- $\alpha$ is considered to be an important pro-inflammatory cytokine, which mediates the degradation of $\mathrm{I} \kappa \mathrm{B} \alpha$ and promotes translocation of NF- $\kappa \mathrm{B}$ to the nucleus (27). Translocation of $\mathrm{NF}-\kappa \mathrm{B}$ activates the transcription of various genes that are involved in cell proliferation, including IL-6 and caspase-3 (28), which were demonstrated to mediate post-liver transplantation intestinal injury amplification and cell apoptosis. Thus, TNF- $\alpha$, IL-6, and caspase- 3 were important in the development of intestinal injury following liver transplantation in the present study. This was demonstrated to be correlated with pathological injury of the intestine. These results suggested that pro-inflammatory cytokine overproduction induced apoptosis following liver transplantation and were critical in post-liver transplantation intestinal injury.

In the present study, it was demonstrated that caspase-3 expression and TUNEL-positive epithelial cells in rats undergoing AOLT were markedly increased compared with that in the sham group (Fig. 4). This suggested that apoptosis was important in AOLT-induced intestinal injury. Caspase-3 was considered to be the executor of apoptosis in the 
caspase cascade and resulted in apoptosis in various types of tissue (29). Many different factors induce apoptosis, such as intestinal congestion-mediated I/R injury, bacterial translocation, oxidative stress or inflammatory reaction (30-32). However, until now, intestinal cell apoptosis resulting from liver transplantation has not been well investigated. Thus, to the best of our knowledge, the present study was the first to investigate possible mechanisms of AOLT-induced intestinal injury. It was demonstrated that intestinal injury was associated with cell apoptosis mediated by endotoxin and cytokine over-production triggered by $\mathrm{NF}-\kappa \mathrm{B}$ signaling pathway activation.

Liver transplantation exhibits serious complications on a number of different systems. The present study, predominantly focused on intestinal injuries and investigated the possible underlying mechanisms. During surgery, IVC and the PV interruption during the anhepatic phrase, resulted in blood reflux disorder, which led to intestinal congestion (3). This impaired intestinal motility and barriers, resulting in an increase of bacterial translocation and overproduction of enterogenous endotoxin (4). Endotoxins may activate the TLR4/NF- $\kappa \mathrm{B}$ signaling pathway, and induce TNF- $\alpha$ production and cell apoptosis. In addition, TNF- $\alpha$ promoted translocation of $\mathrm{NF}-\kappa \mathrm{B}$ to the nucleus mediated by the degradation of $\mathrm{I} \kappa \mathrm{B} \alpha$, resulting in intestinal cell apoptosis and injury (33). Thus, the cascade was amplified intensively and intestinal injury increased.

In conclusion, post-liver transplantation intestinal injury was shown to be associated with TLR4/NF- $\mathrm{B}$ signaling pathway activation-induced cell apoptosis. These findings may aid in the development of effective strategies for intestine protection, as well as protection of other organs, during liver transplantation.

\section{Acknowledgements}

The current study was supported by the National Natural Science Foundation of China (grant nos. 81471892 and 81401628); the Natural Science Foundation of Guangdong Province, China (grant no. S2012010008930); the Science and Technology Planning Project of Guangdong Province, China (grant nos. 2013B021800181 and 2008B030301053); and the Fundamental Research Funds for the Central Universities of China (grant no. 14ykpy24).

\section{References}

1. Luo C, Yuan D, Li X, Yao W, Luo G, Chi X, Li H, Irwin MG, $\mathrm{Xia} Z$ and Hei Z: Propofol attenuated acute kidney injury after orthotopic liver transplantation via inhibiting gap junction composed of connexin 32. Anesthesiology 122: 72-86, 2015.

2. Ohkohchi N: Mechanisms of preservation and ischemic/reperfusion injury in liver transplantation. Transplant Proc 34: 2670-2673, 2002 .

3. Ge M, Chi X, Zhang A, Luo G, Sun G, Xie H and Hei Z: Intestinal NF-E2-related factor-2 expression and antioxidant activity changes in rats undergoing orthotopic liver autotransplantation. Oncol Lett 6: 1307-1312, 2013.

4. Goto S, Kamada N, Moore T, Ware F, Lord R, Kobayashi E and Kim YI: The influence of intestinal congestion on survival of preserved liver grafts in rat liver transplantation. Transplantation 58: 974-977, 1994.

5. Dai X and Wang B: Role of gut barrier function in the pathogenesis of nonalcoholic fatty liver disease. Gastroenterol Res Pract 2015: 287348, 2015.
6. Sanada Y, Mizuta K, Urahashi T, Ihara Y, Wakiya T, Okada N, Yamada N, Ushijima K, Otomo S, Sakamoto K, et al: Impact of endotoxin measured by an endotoxin activity assay during liver transplantation. J Surg Res 180: 349-355, 2013.

7. Li Y, Chen Y, Zhang J, Zhu JF, Liu ZJ, Liang SY, Sun K, Liao WY and Gong JP: Protective effect of glutamine-enriched early enteral nutrition on intestinal mucosal barrier injury after liver transplantation in rats. Am J Surg 199: 35-42, 2010.

8. Luo H, Guo P and Zhou Q: Role of TLR4/NF- $x$ B in damage to intestinal mucosa barrier function and bacterial translocation in rats exposed to hypoxia. PloS One 7: e46291, 2012.

9. Fang WF, Douglas IS, Wang CC, Kao HC, Chang YT, Tseng CC, Huang KT, Chang HC and Lin MC: 5-Lipoxygenase activating protein (FLAP) dependent leukotriene biosynthesis inhibition (MK591) attenuates lipid A endotoxin-induced inflammation. PloS One 9: e102622, 2014.

10. Sarmiento D, Montorfano I, Cáceres M, Echeverría C, Fernández R, Cabello-Verrugio C, Cerda O, Tapia P and Simon F: Endotoxin-induced vascular endothelial cell migration is dependent on TLR4/NF- $\kappa \mathrm{B}$ pathway, NAD $(\mathrm{P}) \mathrm{H}$ oxidase activation and transient receptor potential melastatin 7 calcium channel activity. Int J Biochem Cell Biol 55: 11-23, 2014.

11. Riehl TE, Foster L and Stenson WF: Hyaluronic acid is radioprotective in the intestine through a TLR4 and COX-2-mediated mechanism. Am J Physiol Gastrointest Liver Physiol 302: G309-G316, 2012.

12. Zhang A, Chi X, Luo G, Hei Z, Xia H, Luo C, Wang Y, Mao X and Xia Z: Mast cell stabilization alleviates acute lung injury after orthotopic autologous liver transplantation in rats by downregulating inflammation. PloS One 8: e75262, 2013.

13. Ohkawara H, Ishibashi T, Sugimoto K, Ikeda K, Ogawa K and Takeishi Y: Membrane type 1-matrix metalloproteinase/Akt signaling axis modulates TNF-alpha-induced procoagulant activity and apoptosis in endothelial cells. PloS One 9: e105697, 2014.

14. Mosbah IB, Zaouali MA, Martel C, Bjaoui M, Abdennebi HB, Hotter G, Brenner C and Roselló-Catafau J: IGL-1 solution reduces endoplasmic reticulum stress and apoptosis in rat liver transplantation. Cell Death Dis 3: e279, 2012.

15. Biancofiore G, Bindi L, Miccoli M, Metelli MR, Panicucci E, Baggiani A and Filipponi F: Balance of pro- and anti-inflammatory cytokines in cirrhotic patients undergoing liver transplantation. Transpl Immunol 28: 193-197, 2013.

16. Liboredo JC, Vilela EG, Ferrari MD, Lima AS and Correia MI: Nutrition status and intestinal permeability in patients eligible for liver transplantation. JPEN J Parenter Enteral Nutr 39: 163-170, 2015.

17. Yang S, Li R, Qu X, Tang L, Ge G, Fang W, Qiao Z, Ma J, Hou Y and Liu H: Fosinoprilat alleviates lipopolysaccharide (LPS)-induced inflammation by inhibiting TLR4/NF- $\mathrm{B}$ signaling in monocytes. Cell Immunol 284: 182-186, 2013.

18. Li PM, Li YL, Liu B, Wang WJ, Wang YZ and Li Z: Curcumin inhibits MHCC97H liver cancer cells by activating OS/TLR-4/caspase signaling pathway. Asian Pac J Cancer Prev 15: 2329-2334, 2014.

19. Jung DY, Lee H, Jung BY, Ock J, Lee MS, Lee WH and Suk K: TLR4, but not TLR2, signals autoregulatory apoptosis of cultured microglia: A critical role of IFN-beta as a decision maker. J Immunol 174: 6467-6476, 2005.

20. Zheyu $C$ and Lunan Y: Early changes of small intestine function in rats after liver transplantation. Transplant Proc 38: 1564-1568, 2006.

21. Swank GM and Deitch EA: Role of the gut in multiple organ failure: Bacterial translocation and permeability changes. World J Surg 20: 411-417, 1996.

22. Bedirli A, Sakrak O, Soyuer I and Muhtaroglu S: Portosystemic shunt prevents apoptosis in rat intestinal mucosa caused by total hepatic ischemia. Eur Surg Res 36: 293-299, 2004

23. Zhou QQ, Yang DZ, Luo YJ, Li SZ, Liu FY and Wang GS: Over-starvation aggravates intestinal injury and promotes bacterial and endotoxin translocation under high-altitude hypoxic environment. World J Gastroenterol 17: 1584-1593, 2011.

24. Tanaka KA, Kurihara S, Shibakusa T, Chiba Y and Mikami T: Cystine improves survival rates in a LPS-induced sepsis mouse model. Clin Nutr pii: S0261-S5614, 2014.

25. Guo L, Zheng Z, Ai J, Huang B and Li XA: Hepatic scavenger receptor BI protects against polymicrobial-induced sepsis through promoting LPS clearance in mice. J Biol Chem 289: 14666-14673, 2014. 
26. Huang RL, Yuan Y,Zou GM,Liu G, Tu J and Li Q: LPS-stimulated inflammatory environment inhibits BMP-2-induced osteoblastic differentiation through crosstalk between TLR4/MyD88/NF- $\kappa \mathrm{B}$ and BMP/Smad signaling. Stem Cells Dev 23: 277-289, 2014.

27. Ren Z, Cui J, Huo Z, Xue J, Cui H, Luo B, Jiang L and Yang R: Cordycepin suppresses TNF- $\alpha$-induced $N F-\kappa B$ activation by reducing p 65 transcriptional activity, inhibiting $\mathrm{I} \kappa \mathrm{B} \alpha$ phosphorylation and blocking IKK $\gamma$ ubiquitination. Int Immunopharmacol 14: 698-703, 2012.

28. Huang L, Tang Y, Qin J, Peng Y, Yuan Q, Zhang F and Tao L: Vasoactive intestinal peptide enhances TNF- $\alpha$-induced IL- 6 and IL-8 synthesis in human proximal renal tubular epithelial cells by NF- $\kappa B$-dependent mechanism. Inflammation 35: 1154-1160, 2012

29. Zhang Z, Chen J, Chen L, Yang X, Zhong H, Qi X, Bi Y and $\mathrm{Xu} \mathrm{K}$ : Low frequency and intensity ultrasound induces apoptosis of brain glioma in rats mediated by caspase- $3, \mathrm{Bcl}-2$ and survivin. Brain Res 1473: 25-34, 2012.
30. Dai XJ, Li N, Yu L, Chen ZY, Hua R, Qin X and Zhang YM: Activation of BV2 microglia by lipopolysaccharide triggers an inflammatory reaction in PC12 cell apoptosis through a toll-like receptor 4-dependent pathway. Cell Stress Chaperones 20: 321-331, 2015.

31. Zhou Y, Wang Q, Mark Evers B and Chung DH: Oxidative stress-induced intestinal epithelial cell apoptosis is mediated by p38 MAPK. Biochem Biophys Res Commun 350: 860-865, 2006.

32. Kim JM, Eckmann L, Savidge TC, Lowe DC, Witthöft T and Kagnoff MF: Apoptosis of human intestinal epithelial cells after bacterial invasion. J Clin Invest 102: 1815-1823, 1998.

33. Ahn KS, Sethi G and Aggarwal BB: Simvastatin potentiates TNF-alpha-induced apoptosis through the down-regulation of NF-kappaB-dependent antiapoptotic gene products: Role of IkappaBalpha kinase and TGF-beta-activated kinase-1. J Immunol 178: 2507-2516, 2007. 